\title{
LA INNOVACIÓN COMO EJE DE TRANSFORMACIÓN Y DE CONSTRUCCIÓN SOCIAL Y ECONÓMICA
}

\author{
Mayor GERSON RICARDO JAIMES PARADA ${ }^{1}$
}

Partiendo del hecho, que las especies que sobreviven a la selección natural, son aquellas que mejor se adaptan a los cambios, ya que todos los seres orgánicos se esfuerzan por ocupar todos los puestos en la economía de la naturaleza (DARWIN, 1859), es importante comprender que tanto personas como organizaciones deben estar dispuestas a entender los nuevos contextos y transformase o de lo contrario no estarán a la altura tanto de las nuevas necesidades, como de los retos; los cuales pueden ser locales, regionales y/o mundiales.

Este capítulo pretende hacer una invitación a reflexionar, sobre lo fundamental y la importancia que tiene el hecho de estar alertas a las continuas transformaciones de un mundo globalizado, donde cada día aparecen nuevas oportunidades en diferentes campos de conocimiento, el surgimiento de avances tecnológicos y modelos de negocio de impacto mundial. Todos estos nuevos conocimientos, avances tecnológicos y nuevos modelos de negocio con innovaciones incrementales o disruptivas, van cambiando y mejorando la vida cotidiana y el entorno de familia, que pueden contribuir con la generación o creación de nuevas organizaciones y/o comunidades inteligentes, que afectan directamente estructuras económicas, sociales, antropológicas de cada cultura y País.

La definición de innovación no es tarea fácil, debido a que esta puede cambiar en cada persona de acuerdo a su área de desempeño, experiencia y nivel de formación; por lo anterior es posible encontrar diferentes definiciones. Sin embargo, para propósitos del presente capítulo, la definición más aceptada es la establecida por la Organización de Cooperación y Desarrollo Económico (OECD) en el manual de Oslo que dice:

"una innovación es la introducción de un nuevo, o significativamente mejorado producto (bien o servicio), de un proceso, de un nuevo método de comercialización

Licenciado en Matemáticas y Computación-Universidad Francisco de Paula Santander. Maestría en Tecnologías de la Información Aplicadas a la Educación-Universidad Pedagógica Nacional. Magister en Innovación - Universidad EAN. Subdirector de Investigación-Fuerza Aérea Colombiana. 
o de un nuevo método organizativo, en las prácticas internas de la empresa, la organización del lugar de trabajo o las relaciones exteriores" (OECD, 2005).

Así mismo, el manual de Oslo define cuatro tipos de innovación a saber:

1. Innovación de producto: corresponde con la introducción en la empresa u organización de un bien o servicio nuevo, o significativamente mejorado, en cuanto a sus características o en cuanto al uso al que es destinado (OECD, 2005).

2. Innovación de proceso: Es la introducción en la empresa u organización de un nuevo o significativamente mejorado método de producción o distribución. Esta definición implica cambios sustanciales en las técnicas, equipos y/o los programas de software. Sin embargo, hay que tener cuidado con lo que no son innovaciones de proceso, se refiere a cambios o mejoras menores, el aumento de una capacidad de producción o servicio por la incorporación de sistemas de fabricación o sistemas logísticos que son muy similares a los que ya estén en uso, en la organización o la empresa (OECD, 2005).

3. Innovación de mercadotecnia: Es la introducción de un nuevo método de comercialización que implica cambios significativos del diseño o envasado, de la colocación, la promoción o la tarificación de un producto, siempre y cuando sea la primera vez que se hace en la organización o la empresa (OECD, 2005).

4. Innovación organizativa: Es la introducción de un nuevo método organizativo en las prácticas, la organización del lugar de trabajo o las relaciones exteriores, siempre y cuando sea utilizado por primera vez en la empresa u organización (OECD, 2005).

Es común tener dificultad en identificar que son innovaciones organizativas, por eso, se presentan a continuación algunas situaciones explicadas en el manual de Oslo para contribuir con la aclaración de este tipo de concepto de innovación organizativa:

No se consideran como innovaciones organizativas

- Cuando se utilizan métodos organizativos ya existentes en la empresa u organización, para hacer cambios en las prácticas comerciales, la organización del lugar de trabajo o relaciones exteriores.

- Los cambios en la estrategia de gestión, salvo si son acompañados de la introducción de un nuevo método de organización.

- Fusionar o adquirir nuevas empresas.

- Sí se consideró como innovación organizativa 
- En prácticas empresariales

* La creación de una nueva base de datos sobre las buenas prácticas, lecciones aprendidas y otras formas de generación de conocimiento, para hacerlo de más fácil acceso a terceros.

* Introducción, por primera vez, de un sistema de monitorización integrado de las actividades de una empresa u organización, por ejemplo: producción, finanzas, estrategia, mercadotecnia, gestión de calidad, gestión de $\mathrm{I}+\mathrm{D}+\mathrm{i}$, entre otras.

* Introducción por primera vez, de programas de formación destinados a crear equipos interdisciplinarios y funcionales que integran empleados que vienen de áreas, horizontes o ámbitos con responsabilidades y roles diferentes.

- Organización del lugar de trabajo

* Primera aplicación de una descentralización de las responsabilidades inherentes a sus puestos a los empleados de la empresa u organización, por ejemplo, dando un mayor control y una responsabilidad significativamente mayor sobre el proceso de producción al personal de producción, distribución o ventas.

* Creación por primera vez de equipos de trabajo formales o informales para mejorar la generación, el acceso y la distribución de conocimientos que provienen de diferentes áreas o ámbitos de la empresa u organización, por ejemplo, servicios, producción, investigación, mercadotecnia, entre otros.

* Introducción por primera vez de un sistema de notificación anónimo de incidentes, errores y riesgos que contribuya con la determinación de sus causas y disminuir su frecuencia o materialización.

- Relaciones exteriores

* Introducción de normas de control para proveedores y subcontratistas.

* Recurrir por primera vez a la externalización de la investigación o producción.

* Primera iniciativa de colaboración en actividades de Ciencia, Tecnología e Innovación con entidades de la triada Universidad, Empresa y Estado.

Así mismo, en el campo del desarrollo de la industria se han definido dos categorías de innovación, partiendo de su efecto en la literatura existente y su impacto sobre el estado de la técnica; estas dos categoría se conocen como innovación incremental y la innovación radical, las cuales se explican a continuación: 
Innovación incremental: se puede entender como la que contempla cambios menores en la base tecnológica de un producto o servicio, amparándose en el conocimiento, la experiencia y las capacidades existentes en la empresa (ZIRGER \& HARTLEY, 1994).

Innovación radical: se caracteriza porque tiene una nueva base tecnológica o una experiencia novedosa de utilidad para los clientes (Ali, 1994), se puede complementar la definición de innovación radical expresando que tiene un alto nivel de complejidad e incertidumbre, que contribuye con el aumento de la necesidad del aprendizaje, la flexibilidad y la adaptabilidad, todo con el fin de afianzar y lograr una ventaja competitiva y beneficios que sean sostenibles en el tiempo (Salavou, 2004).

La siguiente tabla, se presenta para dar una mayor claridad en la asociación que por lo general desde el desarrollo tecnológico, se hace de las dos categorías de innovación (incremental o radical), en los tipos de innovación de producto, servicio, mercadotecnia o proceso, utilizando los conceptos y características de propiedad industrial aceptados para modelos de utilidad y patentes de invención.

Tabla No 1. Comparación de categorías de innovación con patentes de invención y modelos de utilidad

\begin{tabular}{|c|c|}
\hline CATEGORÍA DE INNOVACIÓN & PROPIEDAD INDUSTRIAL \\
\hline $\begin{array}{l}\text { Innovación Incremental: contempla } \\
\text { cambios menores en la base tecnológica } \\
\text { de un producto o servicio, amparándose } \\
\text { en el conocimiento, la experiencia y las } \\
\text { capacidades existentes en la empresa } \\
\text { (Zirger \& Hartley, 1994). }\end{array}$ & $\begin{array}{l}\text { Modelo de Utilidad: es toda nueva forma, configuración } \\
\text { o disposición de elementos, de algún artefacto, } \\
\text { herramienta, instrumento, mecanismo u otro objeto } \\
\text { o de alguna parte del mismo (La Comisión de la } \\
\text { Comunidad Andina, 2000), con las siguientes } \\
\text { condiciones: } \\
\text { - Novedad } \\
\text { - Aplicación Industrial }\end{array}$ \\
\hline $\begin{array}{l}\text { Innovación Radical: se caracteriza } \\
\text { porque tiene una nueva base tecnológica } \\
\text { o una experiencia novedosa de utilidad } \\
\text { para los clientes (Ali, 1994). }\end{array}$ & $\begin{array}{l}\text { Patente de Invención: se otorga patentes para las } \\
\text { invenciones, que sean de producto o de procedimiento, } \\
\text { en todos los campos de la tecnología (La Comisión de } \\
\text { la Comunidad Andina, 2000), que cumplan con las } \\
\text { siguientes condiciones: } \\
\text { - Novedad } \\
\text { - Nivel inventivo } \\
\text { - Aplicación industrial }\end{array}$ \\
\hline
\end{tabular}

Fuente: Elaboración propia del autor 
La importancia de los tipos de innovación y categorías de innovación es porque le permiten a los sectores productivos de un País, establecer estrategias para llegar a mercados nacionales e internacionales, contribuyendo con el crecimiento económico, sin embargo, es importante tener la claridad que las empresas y organizaciones no pueden lograr este crecimiento económico de forma aislada y se requiere procesos y actividades de relacionamiento que permitan compartir y unir esfuerzos y capacidades, con el fin de lograr productos y modelos de negocio con valor diferenciador, que sean sostenibles en el tiempo. En el siguiente aparte se presenta la necesidad de la articulación de las entidades de triada universidad, empresa y Estado.

\section{Articulación de la Triada, Universidad Empresa Estado}

En Latinoamérica, existe una gran dificultad para la adopción de nuevos conocimientos y tecnologías para lograr sectores productivos y empresariales con mayor competitividad mundial debido a las falencias que existen entre las interrelaciones y las extra-relaciones de las entidades de la universidad, empresa y Estado, las cuales son dispersas; contrario a lo que sucede en Países desarrollados, donde funcionan de manera más integradas y con buenos resultados las relaciones de este triángulo. Por lo anterior, Latinoamérica requiere fortalecer el triángulo científico-tecnológico, conformado por las entidades de la triada Universidad, Empresa y Estado, se deben generar nuevas capacidades y oportunidades de adopción de nuevos conocimientos y tecnologías, a través, de la construcción de cambios, que contribuyan con la construcción de nuevas estructuras, el fortalecimiento de valores y actitudes que permitan el desarrollo de proceso de innovación, apalancando el aparato productivo y empresarial (SÁBATO \& BOTANA, 1968).

Así mismo, la sociedad es un actor fundamental para la construcción de alianzas exitosas de la triada Universidad, Empresa y Estado, ya que la sociedad conoce sus necesidades y es la primera beneficiada de los resultados exitosos que se logran, como lo explica la siguiente cita:

"un esquema de trabajo con estas virtudes estimularía el desarrollo de una estructura productiva para una mejor inserción y cohesión de los integrantes del desarrollo, con resultados positivos para los agentes de la triple hélice y la sociedad en general" (CAstillo, Lavín, \& Pedraza, 2014).

Los nuevos retos mundiales, exigen que la sociedad colombiana, esté más atenta y tenga un papel más protagónico en las relaciones de la triada Universidad, Empresa y Estado; actualmente se destacan como actores integradores de esta triada, las universidades, los centros de investigación, las oficinas de transferencia de resultados de investigación, las oficinas de transferencia de tecnología, las corporaciones de ciencia, tecnología e innovación y los grupos de investigación.

Sin embargo, se requiere impulsar con mayor apoyo el establecimiento de estas alianzas, con la formación de talento humano de alto nivel y experiencia, y promover 
la generación de conocimiento de alto nivel. De igual forma se requiere inversión en la búsqueda de innovaciones radicales e incrementales, que apalanquen programas académicos de alta calidad en los diferentes niveles académicos de educación superior, que redunden en ventajas competitivas en los sectores productivos nacionales; construyendo así, un tejido económico y social sostenible.

En concordancia con lo anterior, Colombia ha venido incrementando su conocimiento y experiencia en alianza de la triada Universidad Empresa y Estado, para el desarrollo actividades y procesos de gestión de la innovación tecnológica, un actor que ha apoyado este crecimiento, es el Departamento Administrativo de Ciencia, Tecnología e Innovación (COLCIENCIAS); lo cual se evidencia con la creación y fortalecimiento de Oficina de Transferencias de Resultados de Investigación-OTRI (Colciencias, 2013), donde se apoyaron las siguientes:

Tabla No 2. Banco de elegibles para creación o fortalecimiento de OTRI

\begin{tabular}{|l|l|}
\hline \multicolumn{1}{|c|}{ TÍTULO } & ENTIDAD PROPONENTE \\
\hline "OTRI Estratégica del Oriente" & Universidad Autónoma de Bucaramanga \\
\hline $\begin{array}{l}\text { "Creación de una oficina de transferencia de resul- } \\
\text { tados de investigación (OTRI) para la ciudad de } \\
\text { Bogotá como alianza entre la Universidad Distrital } \\
\text { Francisco José de Caldas y la Secretaría Distrital de } \\
\text { Desarrollo Económico" }\end{array}$ & $\begin{array}{l}\text { Universidad Distrital Francisco José } \\
\text { de Caldas }\end{array}$ \\
\hline $\begin{array}{l}\text { "Creación de la oficina de resultados del sector de } \\
\text { seguridad y defensa, basados en las capacidades del } \\
\text { sistema de ciencia y tecnología de la Fuerza Aérea } \\
\text { Colombiana y sus aliados" }\end{array}$ & $\begin{array}{l}\text { Fuerza Aérea Colombiana - Centro de } \\
\text { Ciencia y Tecnología de Antioquia }\end{array}$ \\
\hline $\begin{array}{l}\text { "Creación de una oficina de transferencia de tecnolo- } \\
\text { gía Regional para Bogotá y Cundinamarca" }\end{array}$ & Corporación Connect Bogotá Región \\
\hline $\begin{array}{l}\text { "Fortalecimiento del modelo de transferencia de } \\
\text { tecnología en Antioquia" }\end{array}$ & $\begin{array}{l}\text { Corporación Tecnova Universidad Em- } \\
\text { presa Estado }\end{array}$ \\
\hline $\begin{array}{l}\text { "Diseño y puesta en marcha de una OTRI en el } \\
\text { departamento del Atlántico como estrategia para } \\
\text { dinamizar la transferencia de conocimiento entre } \\
\text { universidad-empresa y sociedad" }\end{array}$ & $\begin{array}{l}\text { Asociación Colombiana de las Micro, } \\
\text { Seccional Atlántico }\end{array}$ \\
\hline
\end{tabular}

Fuente: Colciencias (2013). 
Uno de los aprendizajes más importante en la creación de estas oficinas de transferencia de resultados de investigación, fue la necesidad de unir esfuerzos e impulsar la articulación de la triada Universidad Empresa Estado, con el fin de asimilar metodologías de vigilancia tecnológica e inteligencia competitiva, identificación de mercados potenciales, implementar protocolos para la validación de los resultados y productos de $\mathrm{I}+\mathrm{D}+\mathrm{i}$.

La Fuerza Aérea Colombiana, ha sido un actor importante en este proceso de construcción de Oficinas de Transferencia de Resultados de Investigación, con lo cual ha venido afianzando conocimientos y estrategias para la generación de valor agregado o diferenciador en los productos de $\mathrm{I}+\mathrm{D}+\mathrm{i}$ con potencial comercial, la generación de nuevo conocimiento y la creación de modelos de negocio. De esta forma ha contribuido con la construcción del tejido económico y social del País, por medio del escalamiento industrial y apostando a la comercialización de los productos de $\mathrm{I}+\mathrm{D}+\mathrm{i}$, creando así nuevas formas de emprendimiento, ya sea, a través de licenciamientos, o con la creación o fortalecimiento de spin-off y start-up en diferentes sectores productivos.

Otro aprendizaje relevante de esta experiencia para la creación de OTRI's en Colombia, fue la transmitida por los mentores de la universidad de Austin Texas EEUU y la visita tecnológica realizada al País de Suiza, quienes recalcan la necesidad imperiosa de promover la inversión en actividades de ciencia, tecnología e innovación y la formación de alto nivel, para obtener productos y servicios con valor diferenciador que tengan una buena oportunidad de competitividad y desarrollo empresarial a nivel mundial, y de esta manera lograr mejorar la calidad de vida de la sociedad, teniendo como base un tejido económico y social sostenible.

Finalmente la Fuerza Aérea Colombiana, a través de la Jefatura de Educación Aeronáutica, la Dirección de Ciencia, Tecnología e Innovación a través de sus Escuelas de Formación, Centros y Grupos de Investigación han venido generando y desarrollando políticas y estrategias para incentivar la articulación de la triada Universidad Empresa y Estado, con miras a conseguir resultados de $\mathrm{I}+\mathrm{D}+\mathrm{i}$ de uso dual con valor diferenciador, desde los diferentes campos de conocimiento y aplicación industrial para obtener el impulso de sectores productivos exitosos en beneficio del País.

\section{EPÍLOGO DEL CAPÍTULO}

Globalmente, en este capítulo se invitó al lector a realizar una reflexión sobre la importancia de estar continuamente transformándose tanto a nivel personal, como organizacional y poder responder a los nuevos desafíos que nos ofrece el mundo contemporáneo. En lo referente a transformación personal se puede 
profundizar en temas como la inteligencia emocional, a nivel organizacional para lograr equipos de alto desempeño, se puede profundizar en áreas como la inteligencia social, en ambos casos tienen un componente muy fuerte de la comprensión y administración de las células espejo, que son las que nos permiten tener una mejor o menor empatía con nuestros semejantes.

El hecho de comprender la transformación como una actividad constante nos conducirá a obtener resultados con innovaciones en producto, proceso, servicio u organizativas ya sean incrementales o radicales como se abordó en este capítulo, la Fuerza Aérea Colombiana lo ha comprendido y ha asumido el compromiso de transformarse continuamente, prueba de eso son sus actividades continuas de transformación organizacional donde es un componente vital la innovación, donde se evoluciona constantemente desde los procesos más sencillos de gestión organizacional hasta las tecnologías o nuevos conocimientos más avanzados en el campo de las operaciones áreas.

También se evidencia en la articulación de la Fuerza Aérea Colombiana con el Sistema Nacional de Ciencia Tecnología e Innovación-Colciencias, donde se han logrado actividades en CTeI exitosas, como son la Convocatoria Conjunta para proyectos de I+D+i No 666 en el año 2014, donde se articularon empresas y universidades a nivel nacional para el "Fortalecimiento de los Programas Estratégicos de CTeI de la FAC", así mismo, la creación del modelo de gestión de CTeI en el año 2015, fundamentado en el manual de Oslo, el manual de Frascati y la norma Icontec NTC-5801 que comprende la Gestión de la Investigación, Desarrollo e Innovación (I+D+i) Requisitos del Sistema de Gestión de la I+D+i.

Por último, se tiene proyectado para el año 2018 con la ayuda de los aliados del sector académico y del sector productivo de la FAC, la implementación de un modelo de innovación abierta, que disminuirá debilidades y aumentará capacidades, aplicando el concepto de redes inteligentes, que contribuirá con la articulación en equipos de alto desempeño de las entidades de la triada Universidad-Empresa-Estado, con la generación de nuevo conocimiento, además, obteniendo productos de $\mathrm{I}+\mathrm{D}+\mathrm{i}$ con creación de valor, los cuales contribuirán directamente con la construcción del tejido económico y social nacional.

\section{REFERENCIAS}

Ali, A. (1994). "Pioneering versus incremental innovation: Review and research propositions." Journal of Product Innovation Management, 11(1), 46-56.

Castillo, L., Lavín, J., \& Pedraza, N. (2014). La gestión de la triple hélice: fortaleciendo las relaciones entre la universidad, empresa, gobierno. Multiciencias, 14(4), 438-446. 
Colciencias. (2013). Convocatoria para conformar un banco de propuestas elegibles para la creación o fortalecimiento de oficinas de transferencia de resultados de investigación (OTRI). Retrieved from http://legadoweb. colciencias.gov.co/convocatoria/convocatoria-para-conformar-un-banco-de-propuestas-elegibles-para-la-creaci-n-o-fortale

DARWIN, C. (1859). On the Origin of Species by Means of Natural Selection, or the Preservation of Favoured Races in the Struggle for Life. London: John Murray.

La Comisión de la Comunidad Andina. (2000). Decisión 486:: Régimen Común Sobre Propiedad Industrial. Retrieved from http://www.wipo.int/edocs/ lexdocs/laws/es/can/can012es.pdf

Organización de Cooperación y Desarrollo Económico (OECD), \& OECD. (2005). Manual de Oslo, La Medida de las Actividades Cientificas y Tecnológicas, Guía para la recogida e interpretación de datos sobre innovación. OECD (Vol. 30). http://doi.org/10.1787/9789264065659-es

SÁbAto, J., \& Botana, N. (1968). La ciencia y la tecnología en el desarrollo futuro de América Latina. Revista de La Integración, 1(3), 15-36.

Salavou, H. (2004). The concept of innovativeness: should we need to focus? European Journal of Innovation Management, 7(1), 33-44. http://doi. org/10.1108/14601060410515628

ZIRGER, B. J., \& Hartley, J. L. (1994). A conceptual model of product development cycle time. Journal of Engineering and Technology Management, 11(3-4), 229-251. http://doi.org/10.1016/0923-4748(94)90011-6 